\title{
Performance of Zero-till Wheat (Triticum aestivum L.) and Weed Species as Influenced by Residue and Weed Management Techniques in Rice based Cropping System
}

\author{
Rakesh Kumar $^{1 *}$, U.P. Singh ${ }^{2}$ and Gaurav Mahajan ${ }^{3}$ \\ ${ }^{1}$ Department of Agronomy \& Soil Science, CSIR-CIMAP Research Centre, \\ Pantnagar-263149, India \\ ${ }^{2}$ Department of Agronomy, Banaras Hindu University, Varanasi-221005, India \\ ${ }^{3}$ Department of Agronomy, College of Agriculture, Rewa-486114 (JNKVV), India \\ *Corresponding author
}

\section{A B S T R A C T}

\begin{tabular}{|c|}
\hline Keywords \\
\hline $\begin{array}{l}\text { Residue } \\
\text { management, Weed } \\
\text { control, Zero } \\
\text { tillage, Wheat }\end{array}$ \\
\hline Article Info \\
\hline $\begin{array}{l}\text { Accepted: } \\
\text { 04 March } 2019 \\
\text { Available Online: } \\
10 \text { April } 2019\end{array}$ \\
\hline
\end{tabular}

Keywords

Residue management, Weed tillage, Wheat

\section{Introduction}

Rice (Oryza sativa L.) - Wheat (Triticum aestivum L. emend Fiori \& Poal) cropping sequence is the most predominant production system occupying about 18 Mha in Asia, of which 13.5 Mha area in Indo-Gangetic Plains (IGP) of India (10 Mha), Pakistan (2.2 Mha), Bangladesh (0.8 Mha) and Nepal (0.5 Mha) and feeds about 1.3 billion people (20\% of the world population) (Farooq et al., 2007, Saharawat et al., 2010). This grin scenario is

\begin{abstract}
A field experiment was conducted during 2005-06 to 2006-07 at Varanasi to find out the effect of three residue management practices viz. $R_{1}$ (Residue Removal), $R_{2}$ (Residue Retention alone), $\mathrm{R}_{3}$ (Residue Retention with Trichoderma) and four weed management treatments viz. $\mathrm{W}_{1}$ (Control), $\mathrm{W}_{2}$ (Hand weeding at $30 \& 45 \mathrm{DAS}$ ), $\mathrm{W}_{3}$ (Isoproturon +2 ,4-D $\left(1.0+0.5 \mathrm{ha}^{-1}\right.$ at $\left.30 \mathrm{DAS}\right), \mathrm{W}_{4}$ (Fenoxaprop $120 \mathrm{~g}$ a.i. $\mathrm{ha}^{-1} \mathrm{fb}$ Metsulfuron $4 \mathrm{~g}$ a.i. ha ${ }^{-1}$ ) on weeds and productivity of zero-till wheat (Triticum aestivum L. emend Fiori \& Poal) in rice-wheat cropping system during the winter $(\mathrm{rabi})$ season. Wheat grown under rice wheat cropping system with residue retention with Trichoderma application produced 8.2 and 6.8 $\%$ higher grain and 7.3 and $6.2 \%$ straw yield over residue removal treatment during both the year of experimentations. Out of all residue management approaches under test, residue management alone along with isoproturon + 2, 4-D application for weed control gave higher net returns during the course of above study.
\end{abstract}

stemmed from the exhaustive nature of both the crops because belonging to the same family and their extreme tillage requirement. The new Resource Conservation Technologies (RCTs) develop over the past ten years provide an opportunity to reduce the cost of production. Among these, new RCTs, the zero tillage technologies of wheat cultivation have been adopted over large areas. This technology save more than $90 \%$ energy, time, labour and helps to produce wheat at a much lower cost. Uncontrolled 
weed growth may reduce wheat yield ranging from 15-40 \% depending upon magnitude, nature and duration of weed infestation (Jat et al., 2003). These call for the use of other broad spectrum herbicides either independently or in combination for the management of complex weed flora of wheat to avoid perceptible change in weed flora. The weeds were reduced under zero till sowing in standing stables as rice straw acted as mulch, in partial burning treatment due loss of viability of weed seeds due to high temperature generated during burning and in bed planting due to drying of bed tops which reduced the germination of weeds and hence dry weight was also less under these treatment (Brar and Walia, 2007). Less effort has been made to manage weed population by imposing diverse designed tillage techniques with residue retention. Hence, the present study was, therefore, undertaken to assess the efficacy of herbicides against weeds along with residue retention which have direct effect on weed infestation in wheat crop under the rice- wheat cropping system.

\section{Materials and Methods}

Field trials on wheat crop were conducted at Varanasi (latitude $25^{\circ} 18^{\prime} \mathrm{N}$, longitude $83^{\circ} 03^{\prime} \mathrm{E}$ and altitude $128.93 \mathrm{~m}$ above mean sea level) during 2005-06 to 2006-07. The soil was sandy clay loam, low in available N (200 $\mathrm{kg} / \mathrm{ha})$ medium in organic carbon $(0.44 \%)$, available P (16.2 kg/ha) and available K (240 $\mathrm{kg} / \mathrm{ha})$ with $\mathrm{pH} 7.8$ and EC $(0.19 \mathrm{ds} / \mathrm{m})$. The experimental design was split plot design with thrice replications. Main plot treatments were three residue management techniques viz. $R_{1}$ (Residue Removal), $\mathrm{R}_{2}$ (Residue Retention alone), $\mathrm{R}_{3} \quad$ (Residue Retention with Trichoderma), and sub plot treatments were four weed management techniques viz. $\mathrm{W}_{1}$ (control), $\mathrm{W}_{2}$ (Hand weeding at $30 \& 45$ DAS), $\mathrm{W}_{3}$ [Isoproturon +2, 4-D $(1.0+0.5 \mathrm{~kg}$ $\left.\mathrm{ha}^{-1}\right)$ at 30 Days after sowing], $\mathrm{W}_{4}$
(Fenoxaprop $120 \mathrm{~g} \mathrm{ha}^{-1} \mathrm{fb}$ Metsulfuron $4 \mathrm{~g}$ $\mathrm{ha}^{-1}$ ). During both the years' wheat crop was sown with zero tillage maintaining the rice residue in respective plots as per treatments allocated. Isoproturon + 2, 4-D $(1.0+0.5 \mathrm{~kg}$ $\left.\mathrm{ha}^{-1}\right)$ at $\left.30 \mathrm{DAS}\right)$ and Fenoxaprop (120 $\mathrm{g} \mathrm{ha}^{-1}$ ) $\mathrm{fb}$ Metsulfuron $\left(4 \mathrm{~g} \mathrm{ha}^{-1}\right)$ were applied as post emergence with 500 liters of water with the help of knap sack sprayer, fitted with flat-fan nozzle at 30 DAS. Rice 'Sarjoo-52' was grown from June to October for residue purpose only as a commercial crop and no observations were made and wheat 'HUW234' was grown from November to March in each treatment with recommended package of practices. The experiment was conducted under irrigated conditions. Weed density was recorded (at 45 and at harvest stage) from $0.25 \mathrm{~m}^{-2}$ area by placing a quadrate of $0.5 \times$ $0.5 \mathrm{~m}$ randomly at three places in each plot. A total ten species of weeds enclosed in each quadrate were identified as well as counted species wise and was expressed as number of weeds per square meters.

\section{Results and Discussion}

\section{Effect on weeds}

Weed flora of wheat differ from field to field, depending on environmental conditions, irrigation, fertilizer use, soil type, weed control practices and cropping sequences. The predominant weeds associated with conventional and zero-till wheat are Poa annua, Polypogon monspeliensis, Avena ludoviciana, Rumex dentatus, $R$. spinosus, Anagallis arvensis, Convolvulus arvensis, Malva parviflora, Medicago denticulata, Chenopodium album, Vicia sativa, Lathyrus aphaca, Circium arvense, Melilotus alba, Coronopus didymus, Polygonum plebejum and Spergula arvensis. Among grassy weeds, $P$. minor and among broad-leaved weeds, Rumex dentatus and Medicago denticulata are of major concern in irrigated wheat under 
rice-wheat system in India (Balyan and Malik 2000, Chhokar et al., 2006). Dominant weed species present in the experimental site were Phalaris minor, was grasses, Cyprus rotundous was sedge and other four broad leaf weed species viz. Chenapodium album, Rumex denticulate, Anagalis arvensis and Melilotus species were identified as major one during both the year of investigation. The population of weeds was found to be maximum at $45^{\text {th }}$ day stage (Table 1) and thereafter it decreased at successive stage of crop growth (Table 2) during both the years, irrespective of treatments.

This was owing to death of most of the broad leaved weds which had completed their life cycle before of crop. Residue management resulted in significant reduction in all the weed species as compared to residue removal. Among weed species Phalaris minor showed lower population at 45 DAS in residue retention with Trichoderma application (6.05 $\&$ 5.28) which was at par with residue retention alone (2.41 \& 2.24) and significantly lower to residue removal $(2.56 \&$ 2.44) during both years of investigation. Similarly density of Cyprus rotundous, Chenapodium album, Rumex denticulate, Anagalis arvensis and Melilotus species were found significantly lower population in residue retention with Trichoderma application treatment from residue removal and closely related to residue retention alone. This was due to the covering of soil surface with crop residue and it caused mulching effect during crop period as reported by Kumar et al., (2004), Chhokar et al., (2009) and Kumar et al., (2013). All the weed control methods caused significant reduction in weed density over weedy check. Minimum weed densities were observed under hand weeding twice at $30 \& 45$ DAS. Among the herbicides, application of fenoxaprop $120 \mathrm{~g} \mathrm{ha}^{-1} \mathrm{fb}$ metsulfuron $4 \mathrm{~g} \mathrm{ha}^{-1}$ was showed significant reducing population of all weeds as compared to isoproturon $+2,4-\mathrm{D}\left(1.0+0.5 \mathrm{~kg} \mathrm{ha}^{-1}\right)$ at all stages of growth during both the years except Cyprus rotundas which was resulted at par in 2006-07. It was might be due to higher efficacy of herbicide. Singh and Singh (2002) and Singh et al., (2015) reported that the higher efficacy of Fenoxaprop $120 \mathrm{~g} \mathrm{ha}^{-1} \mathrm{fb}$ Metsulfuron $4 \mathrm{~g} \mathrm{ha}^{-1}$ was due to effective control of narrow as well as broad leaf weeds.

\section{Effect on yield attributes and yield}

Grain yield is considered to be a function of various yield attributing characters like ear length, grains ear head ${ }^{-1}$ and 1000-grain weight. Ear length was significantly influenced by residue management (Table 3). Highest ear length was recorded by residue retention with Trichoderma application (9.19 \& 9.26) followed by residue retention alone $(9.09 \& 9.19)$ and residue removal treatment (8.56 \& 8.71). However, residue retention with Trichoderma application (9.19 \& 9.26) and residue retention alone $(9.09 \& 9.19)$ were statistically at par to each other but they registered significantly higher ear length than residue removal $(8.56 \& 8.71)$ during both the year of investigation.

It might be higher solubility and availability of nutrients to plant through cellulolytic fungous in residue retention with Trichoderma application and thus resulted in better development of yield attributes over other treatments (Singh and Yadav, 2006). In weed management hand weeding twice had the highest ear length (9.25 \& 9.29), grains ear head ${ }^{-1}(47.87 \& 49.31)$ and 1000-grain weight (41.33 \& 41.66) which was at par to fenoxaprop $f b$ metsulfuron and isoproturon + 2, 4-D and these were found significantly superior to weedy check which had the lowest ear length (8.33 \& 8.47), grains ear head ${ }^{-1}$ (41.25 \& 43.34) and 1000-grain weight (38.23 $\&$ 39.33) during both the year of investigations. 
Table.1 Effect of residue and weed management on weed species in zero-till wheat at 45 days after sowing

\begin{tabular}{|c|c|c|c|c|c|c|c|c|c|c|c|c|}
\hline \multirow[t]{2}{*}{ Treatment } & \multicolumn{2}{|c|}{ P. minor } & \multicolumn{2}{|c|}{ C. rotundas } & \multicolumn{2}{|c|}{ C. album } & \multicolumn{2}{|c|}{ A. arvensis } & \multicolumn{2}{|c|}{ R. denticulate } & \multicolumn{2}{|c|}{ Mellilotus spps } \\
\hline & $2005-06$ & 2006-07 & 2005-06 & 2006-07 & 2005-06 & 2006-07 & $2005-06$ & 2006-07 & $2005-06$ & 2006-07 & 2005-06 & 2006-07 \\
\hline \multicolumn{13}{|c|}{ Residue Management } \\
\hline $\mathbf{R}_{1}$ & $\begin{array}{c}2.56 \\
(7.29)\end{array}$ & $\begin{array}{c}2.44 \\
(6.31)\end{array}$ & $\begin{array}{c}2.35 \\
(5.56)\end{array}$ & $\begin{array}{c}2.22 \\
(4.76)\end{array}$ & $\begin{array}{c}2.56 \\
(6.87)\end{array}$ & $\begin{array}{c}2.41 \\
(5.98)\end{array}$ & $\begin{array}{c}2.28 \\
(5.08)\end{array}$ & $\begin{array}{c}2.21 \\
(4.59)\end{array}$ & $\begin{array}{c}2.40 \\
(5.74)\end{array}$ & $\begin{array}{c}2.16 \\
(4.53)\end{array}$ & $\begin{array}{c}2.18 \\
(4.69)\end{array}$ & $\begin{array}{c}2.07 \\
(4.00)\end{array}$ \\
\hline $\mathbf{R}_{2}$ & $\begin{array}{c}2.41 \\
(6.52)\end{array}$ & $\begin{array}{c}2.24 \\
(5.34)\end{array}$ & $\begin{array}{c}2.28 \\
(5.12)\end{array}$ & $\begin{array}{c}2.17 \\
(4.53)\end{array}$ & $\begin{array}{c}2.49 \\
(6.43)\end{array}$ & $\begin{array}{c}2.34 \\
(5.62)\end{array}$ & $2.25(4.90)$ & $\begin{array}{c}2.16 \\
(4.32)\end{array}$ & $\begin{array}{c}2.30 \\
(5.26)\end{array}$ & $\begin{array}{c}2.12 \\
(4.35)\end{array}$ & $\begin{array}{c}2.13 \\
(4.38)\end{array}$ & $\begin{array}{c}2.01 \\
(3.62)\end{array}$ \\
\hline $\mathbf{R}_{3}$ & $\begin{array}{l}2.33 \\
(6.05)\end{array}$ & $\begin{array}{c}2.22 \\
(5.28)\end{array}$ & $\begin{array}{c}2.24 \\
(4.90) \\
\end{array}$ & $\begin{array}{c}2.16 \\
(4.49)\end{array}$ & $\begin{array}{c}2.47 \\
(6.30)\end{array}$ & $\begin{array}{c}2.33 \\
(5.56) \\
\end{array}$ & $2.23(4.81)$ & $\begin{array}{c}2.15 \\
(4.29)\end{array}$ & $\begin{array}{c}2.29 \\
(5.19)\end{array}$ & $\begin{array}{c}2.11 \\
(4.29)\end{array}$ & $\begin{array}{c}2.12 \\
(4.33) \\
\end{array}$ & $\begin{array}{c}1.99 \\
(3.54)\end{array}$ \\
\hline SEm \pm & 0.04 & 0.02 & 0.01 & 0.01 & 0.01 & 0.01 & 0.01 & 0.01 & 0.01 & 0.01 & 0.01 & 0.01 \\
\hline $\mathrm{CD}(P=0.05)$ & 0.11 & 0.05 & 0.04 & 0.04 & 0.04 & 0.05 & 0.03 & 0.04 & 0.04 & 0.04 & 0.04 & 0.04 \\
\hline \multicolumn{13}{|c|}{ Weed Management } \\
\hline $\mathbf{W}_{1}$ & $\begin{array}{c}4.51 \\
(19.38)\end{array}$ & $\begin{array}{c}4.15 \\
(16.21)\end{array}$ & $\begin{array}{c}4.15 \\
(16.23)\end{array}$ & $\begin{array}{c}3.55 \\
(11.59)\end{array}$ & $\begin{array}{c}4.15 \\
(16.23)\end{array}$ & $\begin{array}{c}3.98 \\
(14.84)\end{array}$ & $3.60(12.00)$ & $\begin{array}{c}3.33 \\
(10.08)\end{array}$ & $\begin{array}{c}3.75 \\
(13.05)\end{array}$ & $\begin{array}{c}3.56 \\
(11.71)\end{array}$ & $\begin{array}{c}3.58 \\
(11.82)\end{array}$ & $\begin{array}{c}3.22 \\
(9.35)\end{array}$ \\
\hline $\mathbf{W}_{2}$ & $\begin{array}{c}1.00 \\
(0.00)\end{array}$ & $\begin{array}{c}1.00 \\
(0.00)\end{array}$ & $\begin{array}{c}1.00 \\
(0.00)\end{array}$ & $\begin{array}{c}1.00 \\
(0.00)\end{array}$ & $\begin{array}{c}1.00 \\
(0.00)\end{array}$ & $\begin{array}{c}1.00 \\
(0.00)\end{array}$ & $1.00(0.00)$ & $\begin{array}{c}1.02 \\
(0.03)\end{array}$ & $\begin{array}{c}1.00 \\
(0.00)\end{array}$ & $\begin{array}{c}1.00 \\
(0.00)\end{array}$ & $\begin{array}{c}1.00 \\
(0.00)\end{array}$ & $\begin{array}{c}1.00 \\
(0.00)\end{array}$ \\
\hline $\mathbf{W}_{3}$ & $\begin{array}{c}2.31 \\
(4.38)\end{array}$ & $\begin{array}{c}2.22 \\
(3.96)\end{array}$ & $\begin{array}{c}2.55 \\
(5.51)\end{array}$ & $\begin{array}{c}2.11 \\
(3.44)\end{array}$ & $\begin{array}{c}2.55 \\
(5.51)\end{array}$ & $\begin{array}{c}2.39 \\
(4.72)\end{array}$ & $2.23(3.98)$ & $\begin{array}{c}2.19 \\
(3.80)\end{array}$ & $\begin{array}{c}2.45 \\
(4.98)\end{array}$ & $\begin{array}{c}2.06 \\
(3.24)\end{array}$ & $\begin{array}{c}2.11 \\
(3.45)\end{array}$ & $\begin{array}{c}2.02 \\
(3.07)\end{array}$ \\
\hline $\mathbf{W}_{4}$ & $\begin{array}{c}1.92 \\
(2.71)\end{array}$ & $\begin{array}{c}1.84 \\
(2.39)\end{array}$ & $\begin{array}{c}2.32 \\
(4.39)\end{array}$ & $\begin{array}{c}2.08 \\
(3.35)\end{array}$ & $\begin{array}{c}2.32 \\
(4.39)\end{array}$ & $\begin{array}{c}2.08 \\
(3.32)\end{array}$ & $2.18(3.75)$ & $\begin{array}{c}2.16 \\
(3.68)\end{array}$ & $\begin{array}{c}2.13 \\
(3.56)\end{array}$ & $\begin{array}{c}1.90 \\
(2.61)\end{array}$ & $\begin{array}{c}1.90 \\
(2.59)\end{array}$ & $\begin{array}{c}1.86 \\
(2.44)\end{array}$ \\
\hline SEm \pm & 0.03 & 0.01 & 0.02 & 0.01 & 0.02 & 0.01 & 0.01 & 0.01 & 0.01 & 0.01 & 0.01 & 0.01 \\
\hline $\mathrm{CD}(P=0.05)$ & 0.08 & 0.04 & 0.06 & 0.03 & 0.06 & 0.04 & 0.03 & 0.02 & 0.02 & 0.02 & 0.02 & 0.02 \\
\hline
\end{tabular}

Data transformed to $\sqrt{x}+1$. Figure in parentheses indicate original values.

$\mathrm{R}_{1}$ (Residue Removal), $\mathrm{R}_{2}$ (Residue Retention), $\mathrm{R}_{3}$ (Residue Retention with Trichoderma), $\mathrm{W}_{1}$ (Control), $\mathrm{W}_{2}$ (Hand weeding at 30 \&45 DAS), $\mathrm{W}_{3}$ (Isoproturon $+2,4-\mathrm{D}$

$(1.0+0.5 \mathrm{Kg} / \mathrm{ha})$ at $30 \mathrm{DAS}), \mathrm{W}_{4}$ (Fenoxaprop $120 \mathrm{~g} / \mathrm{ha} f b$ Metsulfuron $4 \mathrm{~g} / \mathrm{ha}$ ).

DAS (Days after sowing).

$f b$ (Followed by one week) 
Table.2 Effect of residue and weed management on weed species in zero-till wheat at harvest

\begin{tabular}{|c|c|c|c|c|c|c|c|c|c|c|c|c|}
\hline \multirow[t]{2}{*}{ Treatment } & \multicolumn{2}{|c|}{ P. minor } & \multicolumn{2}{|c|}{ C. rotundas } & \multicolumn{2}{|c|}{ C. album } & \multicolumn{2}{|c|}{ A. arvensis } & \multicolumn{2}{|c|}{ R. denticulate } & \multicolumn{2}{|c|}{ Mellilotus spps } \\
\hline & $2005-06$ & 2006-07 & $2005-06$ & $2006-07$ & $2005-06$ & $2006-07$ & $2005-06$ & 2006-07 & $2005-06$ & $2006-07$ & $2005-06$ & $2006-07$ \\
\hline \multicolumn{13}{|c|}{ Residue Management } \\
\hline $\mathbf{R}_{1}$ & $\begin{array}{c}2.38 \\
(5.73)\end{array}$ & $2.28(5.15)$ & $\begin{array}{c}2.24 \\
(4.48)\end{array}$ & $\begin{array}{c}2.19 \\
(4.17)\end{array}$ & $\begin{array}{c}2.43 \\
(5.70)\end{array}$ & $\begin{array}{c}2.44 \\
(5.64)\end{array}$ & $\begin{array}{c}2.17 \\
(4.07)\end{array}$ & $\begin{array}{c}2.02 \\
(3.41)\end{array}$ & $\begin{array}{c}2.25 \\
(4.48)\end{array}$ & $\begin{array}{c}2.08 \\
(3.71)\end{array}$ & $\begin{array}{c}2.03 \\
(3.54)\end{array}$ & $\begin{array}{c}2.04 \\
(3.51)\end{array}$ \\
\hline $\mathbf{R}_{\mathbf{2}}$ & $\begin{array}{c}2.23 \\
(4.95)\end{array}$ & $2.19(4.79)$ & $\begin{array}{c}2.16 \\
(4.05)\end{array}$ & $\begin{array}{c}2.10 \\
(3.85)\end{array}$ & $\begin{array}{c}2.37 \\
(5.38)\end{array}$ & $\begin{array}{c}2.37 \\
(5.33)\end{array}$ & $\begin{array}{c}2.10 \\
(3.79)\end{array}$ & $\begin{array}{c}1.98 \\
(3.21)\end{array}$ & $\begin{array}{c}2.22 \\
(4.34)\end{array}$ & $\begin{array}{c}2.03 \\
(3.48)\end{array}$ & $\begin{array}{c}2.00 \\
(3.38)\end{array}$ & $\begin{array}{c}1.97 \\
(3.25)\end{array}$ \\
\hline $\mathbf{R}_{\mathbf{3}}$ & $\begin{array}{c}2.21 \\
(4.87)\end{array}$ & $2.18(4.77)$ & $\begin{array}{c}2.15 \\
(4.00)\end{array}$ & $\begin{array}{c}2.09 \\
(3.80)\end{array}$ & $\begin{array}{c}2.35 \\
(5.29)\end{array}$ & $\begin{array}{c}2.37 \\
(5.29)\end{array}$ & $\begin{array}{c}2.07 \\
(3.67)\end{array}$ & $\begin{array}{c}1.96 \\
(3.15)\end{array}$ & $\begin{array}{c}2.20 \\
(4.24)\end{array}$ & $\begin{array}{c}2.03 \\
(3.47)\end{array}$ & $\begin{array}{c}1.97 \\
(3.31)\end{array}$ & $\begin{array}{c}1.97 \\
(3.23)\end{array}$ \\
\hline SEm \pm & 0.01 & 0.01 & 0.01 & 0.01 & 0.01 & 0.02 & 0.01 & 0.01 & 0.01 & 0.01 & 0.01 & 0.01 \\
\hline $\begin{array}{l}\text { CD } \\
(P=0.05)\end{array}$ & 0.04 & 0.04 & 0.04 & 0.04 & 0.04 & 0.05 & 0.04 & 0.04 & 0.04 & 0.04 & 0.03 & 0.04 \\
\hline \multicolumn{13}{|c|}{ Weed Management } \\
\hline $\mathbf{W}_{1}$ & $\begin{array}{c}3.99 \\
(14.93)\end{array}$ & $\begin{array}{c}3.90 \\
(14.24)\end{array}$ & $\begin{array}{c}3.26 \\
(9.62)\end{array}$ & $\begin{array}{c}3.19 \\
(9.17)\end{array}$ & $\begin{array}{c}3.84 \\
(13.73)\end{array}$ & $\begin{array}{c}3.74 \\
(13.02)\end{array}$ & $\begin{array}{c}3.09 \\
(8.55)\end{array}$ & $\begin{array}{c}2.90 \\
(7.44)\end{array}$ & $\begin{array}{c}3.29 \\
(9.80)\end{array}$ & $\begin{array}{c}3.08 \\
(8.47)\end{array}$ & $\begin{array}{c}3.09 \\
(8.52)\end{array}$ & $\begin{array}{c}3.00 \\
(7.99)\end{array}$ \\
\hline $\mathbf{W}_{2}$ & $\begin{array}{c}1.49 \\
(1.22)\end{array}$ & $1.46(1.13)$ & $\begin{array}{c}1.63 \\
(1.66)\end{array}$ & $\begin{array}{c}1.49 \\
(1.22)\end{array}$ & $\begin{array}{c}1.46 \\
(1.13)\end{array}$ & $\begin{array}{c}1.53 \\
(1.34)\end{array}$ & $\begin{array}{c}1.47 \\
(1.16)\end{array}$ & $\begin{array}{c}1.45 \\
(1.10)\end{array}$ & $\begin{array}{c}1.63 \\
(1.65)\end{array}$ & $\begin{array}{c}1.47 \\
(1.16)\end{array}$ & $\begin{array}{c}1.47 \\
(1.16)\end{array}$ & $\begin{array}{c}1.44 \\
(1.08)\end{array}$ \\
\hline $\mathbf{W}_{3}$ & $\begin{array}{c}1.89 \\
(2.58)\end{array}$ & $1.96(2.85)$ & $\begin{array}{c}2.07 \\
(3.29)\end{array}$ & $\begin{array}{c}2.04 \\
(3.18)\end{array}$ & $\begin{array}{c}2.17 \\
(3.72)\end{array}$ & $\begin{array}{c}2.31 \\
(4.35)\end{array}$ & $\begin{array}{c}2.06 \\
(3.23)\end{array}$ & $\begin{array}{c}1.81 \\
(2.30)\end{array}$ & $\begin{array}{l}12.05 \\
(3.20)\end{array}$ & $\begin{array}{c}1.83 \\
(2.36)\end{array}$ & $\begin{array}{c}1.79 \\
(2.22)\end{array}$ & $\begin{array}{c}1.82 \\
(2.32)\end{array}$ \\
\hline $\mathbf{W}_{4}$ & $\begin{array}{c}1.73 \\
(1.99)\end{array}$ & $1.55(1.40)$ & $\begin{array}{c}1.77 \\
(2.13)\end{array}$ & $\begin{array}{c}1.79 \\
(2.20)\end{array}$ & $\begin{array}{c}2.06 \\
(3.24)\end{array}$ & $\begin{array}{c}1.99 \\
(2.98)\end{array}$ & $\begin{array}{c}1.85 \\
(2.42)\end{array}$ & $\begin{array}{c}1.79 \\
(2.19)\end{array}$ & $\begin{array}{c}1.94 \\
(2.26)\end{array}$ & $\begin{array}{c}1.79 \\
(2.22)\end{array}$ & $\begin{array}{c}1.65 \\
(1.73)\end{array}$ & $\begin{array}{c}1.71 \\
(1.93)\end{array}$ \\
\hline SEm \pm & 0.02 & 0.01 & 0.01 & 0.01 & 0.01 & 0.01 & 0.01 & 0.01 & 0.01 & 0.01 & 0.01 & 0.01 \\
\hline $\begin{array}{l}\text { CD } \\
(P=0.05)\end{array}$ & 0.05 & 0.02 & 0.03 & 0.02 & 0.03 & 0.02 & 0.04 & 0.02 & 0.02 & 0.03 & 0.02 & 0.02 \\
\hline
\end{tabular}

Data transformed to $\sqrt{x}+1$. Figure in parentheses indicate original values

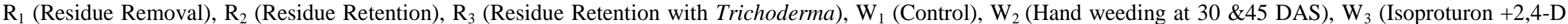
$(1.0+0.5 \mathrm{Kg} / \mathrm{ha})$ at $30 \mathrm{DAS}), \mathrm{W}_{4}$ (Fenoxaprop $120 \mathrm{~g} / \mathrm{ha} \mathrm{fb}$ Metsulfuron $4 \mathrm{~g} / \mathrm{ha}$ )

DAS (Days after sowing)

$f b$ (Followed by one week) 
Table.3 Effect of residue and weed management on Ear length, grains ear head ${ }^{-1}$, test weight, grain and straw yield (kg/ha) in zero-till wheat

\begin{tabular}{|c|c|c|c|c|c|c|c|c|c|c|c|c|}
\hline \multirow[t]{2}{*}{ Treatment } & \multicolumn{2}{|c|}{$\begin{array}{c}\text { Ear length } \\
\quad(\mathrm{cm})\end{array}$} & \multicolumn{2}{|c|}{$\begin{array}{c}\text { Grains ear } \\
\text { head }^{-1}\end{array}$} & \multicolumn{2}{|c|}{ Test weight (g) } & \multicolumn{2}{|c|}{$\begin{array}{l}\text { Grain Yield } \\
\text { (Kg/ha) }\end{array}$} & \multicolumn{2}{|c|}{$\begin{array}{c}\text { Straw Yield } \\
\quad(\mathrm{Kg} / \mathrm{ha})\end{array}$} & \multicolumn{2}{|c|}{$\begin{array}{c}\text { Net return } \\
\left(₹ \text { ha }^{-1}\right)\end{array}$} \\
\hline & $\begin{array}{l}2005- \\
06\end{array}$ & $\begin{array}{c}2006- \\
07\end{array}$ & $\begin{array}{c}2005- \\
06\end{array}$ & $\begin{array}{c}2006- \\
07\end{array}$ & $\begin{array}{c}2005- \\
06\end{array}$ & $\begin{array}{c}2006- \\
07\end{array}$ & $2005-06$ & 2006-07 & $2005-06$ & $2006-07$ & 2005-06 & 2006-07 \\
\hline \multicolumn{13}{|c|}{$\begin{array}{c}\text { Residue } \\
\text { Management }\end{array}$} \\
\hline $\mathbf{R}_{1}$ & 8.56 & 8.71 & 45.64 & 46.76 & 39.87 & 40.05 & 3068.82 & 3385.66 & 4752.40 & 5020.93 & 18794.86 & 28562.07 \\
\hline $\mathbf{R}_{2}$ & 9.09 & 9.19 & 45.83 & 47.57 & 40.50 & 41.22 & 3264.00 & 3580.62 & 5018.88 & 5295.76 & 20619.00 & 30854.00 \\
\hline $\mathbf{R}_{\mathbf{3}}$ & 9.19 & 9.26 & 46.09 & 47.90 & 40.62 & 41.37 & 3320.73 & 3616.48 & 5100.34 & 5332.27 & 20273.78 & 30286.78 \\
\hline SEm \pm & 0.14 & 0.14 & 0.73 & 0.75 & 0.64 & 0.65 & 51.90 & 55.79 & 60.30 & 82.66 & 470.75 & 723.16 \\
\hline $\begin{array}{l}\text { CD } \\
(P=0.05)\end{array}$ & 0.45 & 0.45 & NS & NS & NS & $\mathrm{NS}$ & 163.54 & 175.78 & 190.00 & 260.43 & 1483.26 & NS \\
\hline \multicolumn{13}{|c|}{ Weed Management } \\
\hline $\mathbf{W}_{1}$ & 8.33 & 8.47 & 41.25 & 43.34 & 38.23 & 39.33 & 2788.56 & 2958.35 & 4672.10 & 4813.83 & 17807.96 & 25541.66 \\
\hline $\mathbf{W}_{2}$ & 9.25 & 9.29 & 47.87 & 49.31 & 41.33 & 41.66 & 3414.67 & 3788.12 & 5118.39 & 5426.33 & 19659.43 & 30017.44 \\
\hline $\mathbf{W}_{3}$ & 9.08 & 9.19 & 47.02 & 48.43 & 40.76 & 41.15 & 3313.11 & 3641.60 & 4988.11 & 5292.34 & 21911.4 & 32679.78 \\
\hline $\mathbf{W}_{4}$ & 9.14 & 9.25 & 47.29 & 48.56 & 41.00 & 41.39 & 3355.06 & 3722.27 & 5050.23 & 5332.77 & 20204.72 & 31364.92 \\
\hline SEm \pm & 0.06 & 0.06 & 0.31 & 0.32 & 0.27 & 0.27 & 21.28 & 23.86 & 34.89 & 34.91 & 140.03 & 187.77 \\
\hline $\begin{array}{l}\text { CD } \\
(P=0.05)\end{array}$ & 0.17 & 0.17 & 0.88 & 0.91 & 0.77 & 0.78 & 60.81 & 68.18 & 99.71 & 99.77 & 400.23 & 536.67 \\
\hline
\end{tabular}

$\mathrm{R}_{1}$ (Residue Removal), $\mathrm{R}_{2}$ (Residue Retention), $\mathrm{R}_{3}$ (Residue Retention with Trichoderma), $\mathrm{W}_{1}$ (Control), $\mathrm{W}_{2}$ (Hand weeding at $30 \& 45 \mathrm{DAS}$ ), $\mathrm{W}_{3}$ (Isoproturon $+2,4-\mathrm{D}(1.0+0.5 \mathrm{Kg} / \mathrm{ha})$ at $30 \mathrm{DAS}), \mathrm{W}_{4}$ (Fenoxaprop $120 \mathrm{~g} / \mathrm{ha} \mathrm{fb}$ Metsulfuron 4g/ha)

DAS (Days after sowing)

$f b$ (Followed by one week) 
However, Application of fenoxaprop $120 \mathrm{~g} \mathrm{ha}^{-1}$ $\mathrm{fb}$ metsulfuron $4 \mathrm{~g} \mathrm{ha}^{-1}$ accounted more ear length $(9.14 \& 9.25)$, grains ear head ${ }^{-1}(47.29 \&$ 48.56) and 1000-grain weight (41.00 \& 41.29) which was at par to isoproturon +2 , 4-D (1.0+0.5 kg ha $\left.{ }^{-1}\right)$ ear length (9.08 \& 9.19), grains ear head ${ }^{-1}(47.02 \& 48.43)$ and 1000grain weight ( $40.73 \& 41.15)$ and these were found significantly superior to weedy check (Table 3) which might be due to higher yield attributing characters as a result of low weed population under this treatment. Thomas et al; (2000) were of the opinion that minimum crop weed competition enables the crop to make maximum use of inputs for the formation and development of yield attributes. Residue retention with Trichoderma application registered significantly higher grain $(8.2 \& 6.8$ $\%)$ and straw (7.3 \& $6.2 \%$ ) yield as compared to residue removal treatment (Table 3 ).

The maximum grain and straw yield was recorded under residue retention with Trichoderma application (3320.73 \& 3616.48) and (5100.34 \& 5332.27) followed by residue retention alone (3264.00 \& 3580.62) and $(5018.88 \& 5295.76)$ and residue removal treatment (3068.82 \& 3385.66) and (4752.40 \& 5020.93). However, residue retention with Trichoderma application (3320.73 \& 3616.48) and (5100.34 \& 5332.27) and residue retention alone (3264.00 \& 3580.62) and (5018.88 \& 5295.76) were statistically at par to each other and significantly higher to residue removal (3068.82 \& 3385.66) and (4752.40 \& 5020.93) in respect of grain and straw yield during both the year of investigations. This reduction in grain yield in residue removal was due to poor crop growth and lower value of yield attributes owing to higher weed competition. These findings were supported by Singh and Yadav (2006). Amongst weed management, hand weeding twice recorded maximum grain (3414.67 \& 3788.12) and straw (5118.39 \& 5426.33 ) yield of wheat which was on par with fenoxaprop $f b$ metsulfuron and significantly superior to weedy check during both the years. Study of data further revealed that grain (3355.06 \& 3722.27) and straw (5050.23 \&
533277) yield of fenoxaprop $f b$ metsulfuron was on par with isoproturon $+2,4-\mathrm{D}$ and significantly superior over weedy check during both the years of experimentation. This could be attributed to efficient control of weeds by fenoxaprop $f b$ metsulfuron as evidenced by lowest density of weeds and higher weed suppression efficiency. Similar results have been reported by Jain et al., (2007) and Singh et al., (2010).

\section{Economics}

The practical utility of any weed control measure can be best judged on the basis of net return. The net income was higher with residue retention with Trichoderma application (₹ 20273.78 \& 30286.78) owing to higher yield, and less cost of cultivation. Irrespective of weed management practices, net income were highest (₹ 21911.4 \& 32679.78) with isoproturon +2 , 4-D (1.0+0.5 kg ha $\left.{ }^{-1}\right)$ followed by fenoxaprop $f b$ metsulfuron maily due to lower cost of cultivation. Similar results have been reported by Jain et al., (2007) and Singh et al., (2010).

On the basis of experimental findings, it is concluded that wheat should be sown under residue retention (30-40 $\mathrm{cm})$ along with application of isoproturon+2, 4-D $(1.0+0.5 \mathrm{~kg}$ ha-1) post emergence (30 DAS) for obtaining higher yield and net return under rice-wheat cropping system of India.

\section{References}

Brar, S.S., Kumar, S., Brar, L.S., Walia, S.S. and Kumar, S. (1998). Effect of crop residue management system on the grain yield and efficacy of herbicides in rice-wheat sequence. Indian Journal of Weed Science 30: 39-43.

Brar, A.S. and Walia, U.S. (2007). Influence of planting techniques and weed control treatments on nutrient uptake by Phalaris minor Retz. And broad leaf weeds in wheat (Triticum aestivum). Indian Journal of Weed Science 39 (1 \& 2): 55-61. 
Chhokar, R.S., Singh, S., Sharma, R.K. and Singh, M. (2009). Influence of straw management on Phalaris minor control. Indian Journal of Weed Science 41: 150-156.

Franke, A.C., Singh, S., Mcroberts, N., Nehra, A.S., Godara, S., Malik, R.K. and Marshall, G. (2007). Phalaris minor seed bank studies: Longevity, seedling emergence and seed production as affected by tillage regime. Weed Research 47: 73-83.

Jat, R.S., Nepalia, V. and Chaudhary, P.D. (2003). Influence of herbicides and method of sowing on weed dynamics in wheat (Triticum aestivum L.). Indian Journal of Weed Science 35: 18-20.

Jain, N., Jain, V., Mishra, J.S. and Kewat, M.L. (2007). Effect of tillage packages and herbicides on energy and economics of wheat in transplanted rice-wheat systems. Indian Journal of Agricultural Science 77(3): 174-176.

Kumar, V., Singh, S., Chhokar, R.S., Malik, R.K., Brainard, D.C. and Ladha, J.K. (2013). Weed management strategies to reduce herbicide use in zero-till ricewheat cropping systems of the IndoGangetic Plains. Weed Technology 27: 241-254.

Kumar, S., Pandey, D.S. and Rana, N.S. (2004). Effect of tillage, rice residue and nitrogen management practices on the yield of wheat (Triticum aestivum L.) and chemical properties of soil under rice (Oryza sativa) - wheat (Triticum aestivum L.) system. Indian Journal of Agronomy 49 (4): 223-225.

Saharawat, Y.S., Singh, B., Malik, R.K., Ladha, J.K., Gathala, M., Jat, M.L. and Kumar,
V. (2010). Evaluation of alternative tillage and crop establishment methods in a rice-wheat rotation in NorthWestern IGP. Field Crops Research 116: 260-267.

Singh, G., Singh, V.P. and Singh, M. (2002). Bioefficacy of metsulfuron-methyl in combination with isoproturon for control of grassy and non-grassy weeds in wheat. Indian Journal of Weed Science 34: 9-12.

Singh, R. and Yadav, D.S. (2006). Effect of rice (Oryza sativa) residue and nitrogen on performance of wheat (Triticum aestivum) under rice-wheat cropping system. Indian J. Agron. 55(2): 83-88.

Singh, G., Singh, O.P., Singh, S. and Prasad, K. (2010). Weed management in late sown wheat (Triticum aestivum) after rice (Oryza sativa) in rice-wheat system in rainfed lowland. Indian J. Agron. 51(4): 247-250.

Singh, A.P., Bhullar, M.S., Yadav, R. and Chowdhury, T. (2015). Weed management in zero-till wheat. Indian Journal of Weed Science 47(3): 233239.

Thomas, C.G., Yaduraju, N.T. and Kumar, J. S. (2000). Estimation of yield losses of wheat caused by wild oats (Avena sterilis spp. ludoviciana) competition. Indian Journal of Weed Science 32: 4450 .

Timsina, J. and Conner, D.J. (2001). Productivity and management of ricewheat cropping system: Issues and challenges. Field crop Research 69: 93132.

\section{How to cite this article:}

Rakesh Kumar, U.P. Singh and Gaurav Mahajan. 2019. Performance of Zero-till Wheat (Triticum aestivum L.) and Weed Species as Influenced by Residue and Weed Management Techniques in Rice based Cropping System. Int.J.Curr.Microbiol.App.Sci. 8(04): 270-277. doi: https://doi.org/10.20546/ijcmas.2019.804.030 